\title{
Double phase conjugation
}

\author{
Doruk Engin, Mordechai Segev, ${ }^{*}$ Sergei Orlov, and Amnon Yariv \\ California Institute of Technology, 128-95, Pasadena, California 91125
}

George C. Valley

Hughes Research Laboratories, 3011 Malibu Canyon Road, Malibu, California 90265

Received January 21, 1994; revised manuscript received March 22, 1994

\begin{abstract}
We model the double phase-conjugate mirror (DPCM) as a function of time, the average direction of propagation of the two beams forming the DPCM, and one transverse coordinate. Calculations show that the conjugation fidelity and reflectivity have different dependencies on the photorefractive coupling coefficient times length; the fidelity turns on abruptly with a threshold, whereas the reflectivity increases smoothly. The DPCM behaves as an oscillator at and above threshold: the time required for the reflectivity to reach the steady state dramatically slows down near threshold (like critical slowing down in lasers); above threshold the DPCM is self-sustaining even if the random noise terms used to start the process are set to zero. A decrease in the noise level improves the fidelity but increases the response time. The use of unbalanced input beam ratios results in asymmetric conjugation such that the fidelity obtained on the side of the weaker input beam is significantly reduced. The slowing down diminishes with increasing noise level or unbalanced input intensities.
\end{abstract}

\section{INTRODUCTION}

The photorefractive (PR) double phase-conjugate mirror ${ }^{1}$ (DPCM) is a unique device in which two mutually incoherent beams become phase conjugates of each other. Because the two beams cannot write stationary gratings, their interaction originally puzzled many researchers. Weiss $e t$ al. ${ }^{1}$ first realized that both beams fan and that, because they share the same holographic medium, an effective interaction between them is produced through the scattering off each other's gratings. ${ }^{1}$ The double phase-conjugation process was theoretically addressed initially by qualitative one-dimensional models ${ }^{1-3}$ and later through a series of two-dimensional analyses approximating the DPCM as a single-grating interaction. ${ }^{4}$ This study raises some interesting questions about the oscillatory behavior of the DPCM, but two recent independent studies ${ }^{5,6}$ show that the approximation is inapplicable for the DPCM or other stimulated PR processes. Another theoretical analysis describing the self-pumped phase conjugation of a speckled beam in a PR medium was presented by Lyubomudrov and Shkunov ${ }^{7}$ and was experimentally verified by Mamaev and Shkunov. ${ }^{8}$

Recently we developed a model to calculate the temporal and the spatial behavior of fanning ${ }^{9}$ and of the $\mathrm{DPCM}^{6}$ and predicted a gain threshold for the conjugation fidelity. The gain threshold was also observed experimentally. ${ }^{10}$ Our purpose in this paper is to provide a comprehensive description of the underlying theory, to present additional calculations that characterize the DPCM as an oscillator, and to investigate its behavior under varying parameters (seed level, input beam intensity ratios). In Section 2 the theory is described; two paraxial wave equations for the beams and a first-order differential equation (in time) for the perturbation in the index of refraction are reduced to a system of first-order coupled differential equations for the amplitudes of the plane waves constituting the optical beam. Section 3 contains a brief description of the numerical calculations and the definitions of the figures of merit (conjugation reflectivity, conjugation fidelity, intensity transmission, and respective response times) that we use to characterize the DPCM. In Section 4 we establish the fact that the conjugation fidelity and the conjugation reflectivity show significantly different behavior near threshold. The conjugation fidelity has a relatively sharp jump near threshold, whereas the conjugation reflectivity increases smoothly as a function of gain. The temporal evolution quantities of the two differ as well; the response time of the conjugation reflectivity becomes significantly longer than the response time of the conjugation fidelity near threshold. In Section 5 we present results that point out the oscillatory behavior of the DPCM. Here we find that the response time of the device becomes significantly longer near threshold, indicating the existence of critical slowing down, ${ }^{11}$ a behavior that is observed in many nonlinear systems near a critical point (e.g., for laser oscillation near threshold and for second-order phase transitions near the transition point). The behavior of the DPCM is therefore similar to that of other critical phenomena. We also find that if the gain is above threshold the steady-state reflectivity is independent of the initial seed level and remains unchanged even after the seed level is set to zero. In Section 6 the seed level and the intensity ratio dependencies of the gain threshold are investigated. Here we find that for a low seed level the steady-state fidelity and reflectivity do not vary significantly with seed levels. However, for high seed levels fanning becomes more pronounced, and the calculations show a significant degradation of the phase conjugation. We also find that with an increasing seed level the critical slowing down becomes less pronounced. In this section we also present numerical results that illustrate the effect of unbalanced input beam intensities 
on the DPCM. We show that, although the conjugation reflectivity on the side of the weaker input beam becomes considerably larger with higher intensity ratios, the conjugation fidelity significantly degrades. We also observe that the fidelity response time of the beam emerging on the side of the stronger input beam is significantly faster than the fidelity response time on the opposite side, although the average intensity in the medium is fixed. For larger intensity ratios the fanning of the strong beam becomes more apparent, and the critical slowing down is less pronounced.

\section{THEORETICAL MODEL}

Our theoretical formulation starts with the nonlinear wave equation

$$
\nabla^{2} \mathbf{E}-\mu \varepsilon_{0}\left(n_{b}+\delta n\right)^{2} \frac{\partial^{2} \mathbf{E}}{\partial t^{2}}=0,
$$

where $\mathbf{E}$ is the electric field at the optical wavelength $\lambda$ in vacuum and where $\mu$ and $\varepsilon_{0}$ are the magnetic permeability of the crystal and the electric permeability of vacuum, respectively. The index of refraction is taken as the sum of $n_{b}$ (constant-background refractive index in the crystal) and $\delta n(\mathbf{r}, t)$, where the latter is assumed to be much smaller than the former. In our model we analyze only isotropic scattering; thus the index of refraction is taken to be a scalar. Also, for simplicity, we consider only two spatial dimensions $(x, z)$. Note that the nonlinearity in Eq. (1) arises from the implicit dependence of $\delta n(\mathbf{r}, t)$ on the optical field. Also note that Eq. (1) already includes the usual approximations for slow spatial and temporal variations of the refractive index (for derivation of this equation see, e.g., Ref. 12). In this paper boldface letters correspond to vector quantities.

The electric field consists of two counterpropagating monochromatic beams (with the $z$ axis being the nominal direction of propagation) whose slowly varying amplitudes are $A$ and $B$ :

$$
\begin{aligned}
\mathbf{E}(x, z, t)= & 1 / 2[A(x, z, t) \exp (i k z-i \omega t) \\
& +B(x, z, t) \exp (i k z+i \omega t)+\text { c.c. }] \hat{x},
\end{aligned}
$$

where $\omega$ is the frequency and $k=n_{b} \omega / c$ is the wave number in the crystal and $\hat{x}$ is the unit vector in the transverse direction. The small deviation of the propagation angle from $180^{\circ}$ is accounted for in the slowly varying amplitudes of the beams.

We substitute Eq. (2) into wave equation (1) and separately equate terms with the same exponential factors. We neglect terms proportional to $\delta n^{2}$ (assuming that $\left.2 n_{b}|\delta n| \gg|\delta n|^{2}\right)$ and terms with the time derivatives of the amplitudes (we assume that $A$ and $B$ instantaneously follow the evolution of the PR gratings, which is relatively slow ${ }^{13}$ ), and use the paraxial approximation to obtain two coupled-wave equations for the slowly varying amplitudes:

$$
\begin{gathered}
\frac{\partial A}{\partial z}-\frac{i}{2 k} \frac{\partial^{2} A}{\partial x^{2}}=\frac{i k}{n_{b}} \delta n A, \\
\frac{\partial B}{\partial z}+\frac{i}{2 k} \frac{\partial^{2} B}{\partial x^{2}}=-\frac{i k}{n_{b}} \delta n B .
\end{gathered}
$$

Here absorption is neglected; however, we can easily in- clude it in the model by adding $-\alpha A$ and $\alpha B$ to Eqs. (3) and (4), respectively.

The slowly varying amplitudes are expanded in plane waves about the nominal direction of propagation $z$ :

$$
\begin{aligned}
& A(x, z, t)=\sum_{m} a_{m}(z, t) \exp \left(-i k \varepsilon m x-i k \varepsilon^{2} m^{2} z / 2\right), \\
& B(x, z, t)=\sum_{m} b_{m}(z, t) \exp \left(i k \varepsilon m x+i k \varepsilon^{2} m^{2} z / 2\right),
\end{aligned}
$$

where $\varepsilon$ is the small angular difference between the two adjacent plane waves and $a_{m}$ and $b_{m}$ are the expansion coefficients.

The refractive-index perturbation that yields the DPCM is due to the PR effect. The interference between the plane waves from each beam and its scattered waves form gratings in the crystal that lead to the formation of a space-charge (electric) field by means of charge transport. The space-charge field in turn leads to a perturbation in the index of refraction through the electro-optic effect. Because the PR effect has a relatively slow response time, the crystal can respond to only a time average of the intensity profile. ${ }^{13}$ In our case we assume that the gratings formed by the plane waves of two counterpropagating (mutually incoherent) beams vary faster than the response time of the material and hence do not contribute to the refractive-index perturbation. Therefore $\delta n$ may be expanded as

$$
\begin{aligned}
\delta n(x, z, t)= & \sum_{m, n} \delta n_{m n}(z, t) \exp [-i k(m-n) x \varepsilon \\
& \left.-i k \varepsilon^{2}\left(m^{2}-n^{2}\right) z / 2\right],
\end{aligned}
$$

where $\delta n_{m n}$ are the expansion coefficients. Note that the double sum has the same transverse $(x)$ dependence (apart from a possible phase shift) as the interference pattern between the spatial components of each beam (considered separately), given by Eqs. (5) and (6).

The evolution of these coefficients is governed by the following set of nonlinear differential equations:

$$
\begin{aligned}
\tau_{m n} \frac{\partial \delta n_{m n}}{\partial t} & +\left(1+I_{0} / I_{\mathrm{dark}}\right) \delta n_{m n}(z, t) \\
& =i \gamma_{m n} / I_{\mathrm{dark}}\left[a_{m}(z, t) a_{n}{ }^{*}(z, t)+b_{n}(z, t) b_{m}{ }^{*}(z, t)\right]
\end{aligned}
$$

where $I_{0}=\sum_{m} a_{m} a_{m}{ }^{*}+b_{m} b_{m}{ }^{*}$ and $\tau_{m n}=\tau_{\text {dark }}\left(1+\tau_{\mathrm{Re}} /\right.$ $\left.\tau_{D m n}\right) /\left(1+k_{m n}{ }^{2} / k_{d}{ }^{2}\right)$. Here $k_{d}$ and $k_{m n}$ are the Debye wave number and the wave number of the particular grating $\left(\delta n_{m n}\right)$, respectively. The time constants $\tau_{\mathrm{Re}}$ and $\tau_{D m n}$ are the electron-recombination time and the diffusion time, and $I_{\text {dark }}$ is the dark irradiance. The spatial average of $\left(|A|^{2}+|B|^{2}\right)$ in the transverse direction $(x)$ is equal to $I_{0}$, which is a constant in the absence of absorption. The coupling constants between the individual plane waves are $\gamma_{m n}$, which depend on the particular crystal parameters, the angles of propagation, and the polarization of the beams. (See Appendix A for references and a discussion of the derivation of these equations.) In the absence of absorption gratings $\delta n$ is real, and the coupling constants satisfy the relation $\gamma_{n m}=-\gamma_{m n}{ }^{*}$ (which comes from energy conservation). Note that $\gamma_{m n}$ values form an anti-Hermitian matrix. In our case we assume that 
externally applied fields and photovoltaic effects are absent; therefore $\gamma_{n m}$ are real, and $\gamma_{m m}=0$. From Eq. (8), together with the initial values $\delta n_{m n}=0$, one can show that the relations $\delta n_{m n}=\delta n_{n m}{ }^{*}$ and $\delta n_{m m}=0$ hold at all times and for all $m$ and $n$. These relations result in energy-exchange interactions between the spatial components, with no nonlinear phase coupling.

Note that Eq. (8) is valid when the following inequality is satisfied:

$$
\begin{aligned}
\sum_{m} a_{m} a_{m}{ }^{*}+b_{m} b_{m}{ }^{*} \gg & \sum_{m, n}\left\{a_{m} a_{n}^{*} \exp [-i k(m-n) x \varepsilon\right. \\
& \left.-i k \varepsilon^{2}\left(m^{2}-n^{2}\right) z / 2\right]+b_{m} b_{n}{ }^{*} \\
& \times \exp [i k(m-n) x \varepsilon \\
& \left.\left.+i k \varepsilon^{2}\left(m^{2}-n^{2}\right) z / 2\right]\right\} .
\end{aligned}
$$

Inequality (9) implies that the total local intensity [at a given point $(x, z)$ ] does not significantly differ from its spatial average (or, equivalently, that this is the limit of low modulation depth for each grating in the collection of gratings). If the beams have Gaussian spatial profiles, the validity of Eq. (8) becomes questionable at the margins of each beam but holds elsewhere. Hence, for a strongly focused beam [when the beam diameter is of the order of $2 \pi / k \varepsilon(m-n) x]$, the above inequality is not satisfied.

We transform the nonlinear paraxial wave equations (3) and (4) into the following equations for the coefficients $a_{m}, b_{m}$, and $\delta n_{m n}$ :

$$
\begin{aligned}
\frac{\partial a_{p}}{\partial z} & =\frac{i k}{n_{b}} \sum_{m, n} \delta n_{m n} a_{p+n-m} \exp \left(-i k \varepsilon^{2} \Delta_{1} z / 2\right), \\
\frac{\partial b_{p}}{\partial z} & =\frac{-i k}{n_{b}} \sum_{m, n} \delta n_{m n} b_{p-n+m} \exp \left(i k \varepsilon^{2} \Delta_{2} z / 2\right),
\end{aligned}
$$

where $\Delta_{1}=2(m-n)(m-p), \Delta_{2}=2(n-p)(n-m)$.

To derive Eqs. (10) and (11), we substitute the expansions (5)-(7) into the wave equations (3) and (4). This substitution gives two complicated, but similar, equations. Equation (3) becomes

$$
\begin{aligned}
& 2 i k \sum_{m} \frac{\partial a_{m}}{\partial z} \exp \left[-i k(\varepsilon m)^{2} z / 2\right] \exp (-i k m \varepsilon x) \\
&=-2 k^{2} / n_{b} \sum_{m, n, l} a_{m} \delta n_{n l} \exp \left[-i k \varepsilon^{2}\left(m^{2}+n^{2}-l^{2}\right) z / 2\right] \\
& \times \exp [-i k \varepsilon(m+n-l) x] .
\end{aligned}
$$

We obtain the other equation (for $b_{m}$ ) by conjugating Eq. (12) and replacing $a_{m}{ }^{*}$ with $b_{m}$. We then multiply Eq. (12) by $\exp (i k \varepsilon p x)$ and the equation for $b_{m}$ by $\exp (-i k \varepsilon p x)$ and integrate over $x$ (from $-\infty$ to $\infty$ ). This calculation results in delta functions that transform the two equations into Eqs. (10) and (11). The limits of the above integration identify the limits of transverse space (in practice, we assume that the spatial extent of the beams is much smaller than the crystal's width).

The condition $\Delta_{j}=0(j=1,2)$ identifies the phasematched terms in the double sums of Eqs. (10) and (11). The non-phase-matched terms vary rapidly with $z$ (of the order of the optical wavelength), contributing very little to the integration over $z$. In Ref. 9 we performed numerical calculations investigating the influence of these terms on fanning. The results showed that the nonphase-matched terms are small and can be neglected for propagation paths greater than a few hundred micrometers. Therefore we neglect the non-phase-matched terms in the calculations below. Each of the phasematching conditions has two solutions. The terms that correspond to $(m=n)$ are identically zero at all times [because $\delta n_{m m}(z, t)=0$ ]; thus the only contributing (phasematched) terms are those with $m=p$ and $n=p$ in Eqs. (10) and (11), respectively. This results in

$$
\begin{aligned}
& \frac{\partial a_{p}(z, t)}{\partial z}=\frac{i k}{n_{b}} \sum_{n} \delta n_{p n}(z, t) a_{n}(z, t), \\
& \frac{\partial b_{p}(z, t)}{\partial z}=\frac{-i k}{n_{b}} \sum_{n} \delta n_{n p}(z, t) b_{n}(z, t) .
\end{aligned}
$$

Equations (8), (13), and (14) are the final equations of our formulation. This system of differential equations, together with the appropriate boundary conditions and initial values, makes up our mathematical model for DPCM. The boundary conditions and the initial values for $a_{m}$ and $b_{m}$ are arbitrary and correspond to the spatial profiles of the beams. However, they satisfy the relations $a_{m}(z, 0)=a_{m}(0, t)$ and $b_{m}(z, 0)=b_{m}(L, t)$, where $z=0$ is the input plane of beam $\mathrm{A}$ and $z=L$ is the input plane of beam $B$ (input beam profiles are stationary at all times, unless explicitly specified otherwise). The initial values for the coefficients of the perturbation index are $\delta n_{p n}=0$.

Using Eqs. (8), (12), and (13) and the relations $\delta n_{m n}=$ $\delta n_{n m}{ }^{*}, \delta n_{m m}=0$, one can easily show that $\sum_{m}\left|a_{m}\right|^{2}$ and $\sum_{m}\left|b_{m}\right|^{2}$, and $\sum_{m} a_{m} b_{m}$ do not depend on $z$. Physically, $\sum_{m}\left|a_{m}\right|^{2}$ and $\sum_{m}\left|b_{m}\right|^{2}$ correspond to the total intensities of the mutually incoherent beams (which are constants in the absence of absorption), and $\sum_{m} a_{m} b_{m}$ is the spatial correlation (in the transverse dimension) of the two beams. Note that in Section 3 we use the conjugation fidelity [Eq. (18)], which is proportional to $\sum_{m} a_{m} b_{m}$, as a measure of the fidelity of the conjugation. Similar integration constants were found in the one-dimensional theory for the transmission-grating geometry of four-wave mixing. ${ }^{14}$

The DPCM starts from noise that is generated by scattering of the pump beams from inhomogeneities, defects, inclusions, etc. Because the sources of the initial scattering are thought to be stationary, the seeding noise is taken to be independent of time. In Ref. 9 it is shown theoretically that only the scattering at, or near, the input surface is relevant for seeding the fanning. Because the DPCM is initiated by fanning, we assume that the volume scattering may be neglected. The surface scattering is included in the model through the boundary conditions on the two beams, whereby each beam has an angular width of approximately $2^{\circ}$ that is added to a uniform noise level (seed level), which is spread over the entire angular spectrum $\left(10^{\circ}\right.$ in our calculations).

\section{BASIC PROPERTIES OF THE DPCM}

In this section we present some basic characteristics of our numerical results and define the figures of merit that are used to evaluate the level of phase conjugation.

Equations (8), (13), and (14), along with the boundary conditions and the initial values, are solved numerically 


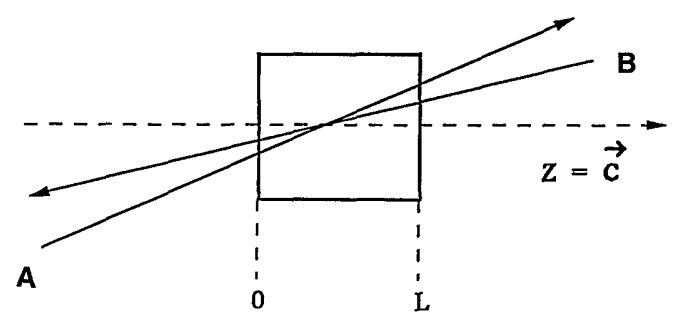

Fig. 1. Sketch of the DPCM.

by the split-step method. Note that in solving these equations we assume that $I_{0}$ is much larger than $I_{\text {dark }}$; hence $1+I_{0} / I_{\text {dark }} \approx I_{0} / I_{\text {dark }}$ in Eq. (8). We use 21 plane waves separated by $0.5^{\circ}$ covering a total angular spectrum of $10^{\circ}$. We verify the validity of our results by doubling the number of integration steps in time and space and by doubling the number of plane waves (for the same angular spectrum; see Appendix B for a discussion of the numerical tests of the calculations). Our calculations involve no Fourier transforms, so the question of aliasing does not arise. In general, scattering from small-scale refractiveindex inhomogeneities results in plane-wave amplitudes outside the finite angular spectrum of our calculations, but for high conjugation quality these amplitudes remain small. In cases with low conjugation fidelity, we discard results in which the plane-wave amplitudes at the edges of the angular spectrum become comparable with those of the beams.

Figure 1 shows the configuration of the DPCM device and the orientations of the beams and the crystal. Note that, in agreement with the usual experimental conditions, the $z$ axis is not parallel to the $c$ axis (we used an angular separation of $12^{\circ}$ between the two axes) to produce a higher gain. ${ }^{15}$ Figure 2 illustrates the steadystate far-field amplitudes of the two beams as functions of the propagation direction $z$. In Fig. 2 (and in Fig. 3 below), all the angles are measured from the forward $c$ axis. At the input plane ( $z=0$ for beam $\mathrm{A}$ and $z=L$ for beam $B$ ), each beam consists of five plane waves (spanning an angular width of $2^{\circ}$ ) with an average amplitude of approximately 1 . The remaining plane waves are taken to be white noise with constant amplitude $\left(10^{-3}\right)$ and pseudorandom phase (between $\pi$ and $-\pi$ ). We refer to the five plane waves that initially make up the image borne on each beam as $\Omega_{A}$ and $\Omega_{B}$ (for beams $A$ and $B$, respectively). In Fig. 2, the input beams are shown on the top right $\left(\Omega_{B}\right)$ and on the bottom left $\left(\Omega_{A}\right)$, and the arrows indicate the directions of propagation for the two beams. As each beam propagates through the crystal, it diffracts and evolves into the phase conjugate of the other beam (top left and bottom right). We refer to the plane waves that make up the phase-conjugate beams as $\Delta_{A}$ and $\Delta_{B}$ (phase conjugates of beam $A$ and $B$, respectively). Figure 3 shows the phase profiles of the steady-state input and output beams at $z=0$ and $z=L$ (only plane waves that belong to the image portions of the beams, i.e., $\Omega_{A}$, $\Omega_{B}, \Delta_{A}, \Delta_{B}$, are shown). Note that the phase-reversed image acquires an additional bias phase (in Fig. 3 this phase is approximately $10^{\circ}$ ), which was also observed experimentally. ${ }^{16}$

To evaluate the level of phase conjugation in the DPCM, we use the following figures of merit:

$$
\begin{aligned}
T_{B}(t) & =\frac{\sum_{i \in \Delta_{A}}\left|b_{i}(0, t)\right|^{2}}{\sum_{i \in \Omega_{B}}\left|b_{i}(L, 0)\right|^{2}}, \\
\mathrm{CR}_{B}(t) & =\frac{\sum_{i \in \Delta_{B}}\left|a_{i}(L, t)\right|^{2}}{\sum_{i \in \Omega_{B}}\left|b_{i}(L, 0)\right|^{2}}, \\
\mathrm{CF}_{B}(t) & =\frac{\sum_{\in\left(i \Omega_{B} \cup \Delta_{B}\right)} a_{i}(L, t) b_{i}(L)}{\left[\sum_{i \in \Delta_{B}}\left|a_{i}(L, t)\right|^{2} \sum_{i \in \Omega_{B}}\left|b_{i}(L)\right|^{2}\right]^{1 / 2}},
\end{aligned}
$$

where $T_{B}, \mathrm{CR}_{b}$, and $\mathrm{CF}_{b}$ are the intensity transmission, the conjugate reflectivity, and the conjugate fidelity of beam $B$, respectively. The sums are taken over the sets of plane waves $\Omega_{A}, \Omega_{B}, \Delta_{A}, \Delta_{B}$, which are defined in the previous paragraph and are illustrated in Figs. 2 and 3 . Note that $a_{i}$ and $b_{i}$ correspond to counterpropagating plane-wave components. The conjugation reflectivity

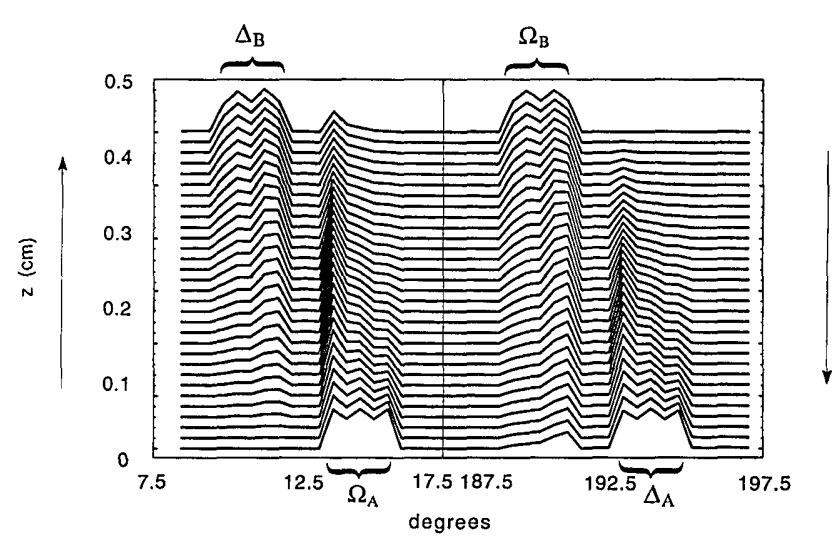

Fig. 2. Steady-state far-field patterns of the two beams as a function of angle. The angles are measured from the forward $c$ axis.

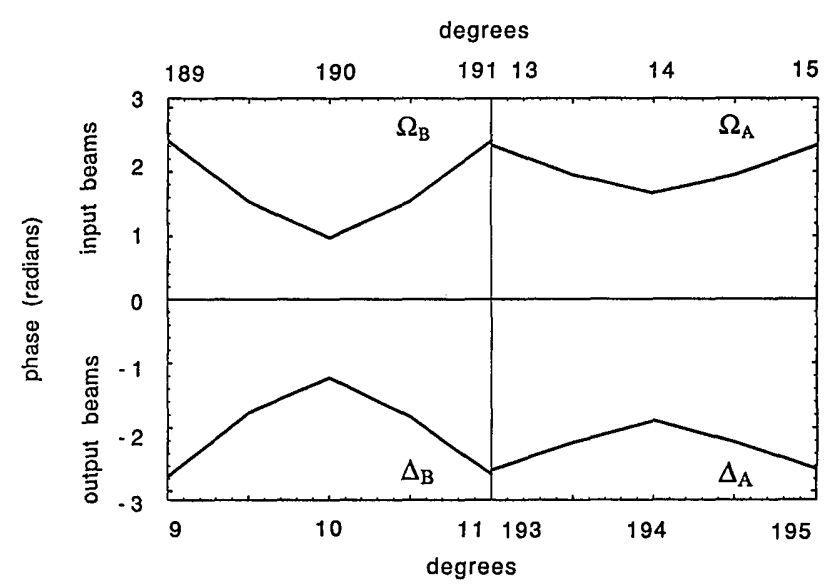

Fig. 3. Phase profiles of the two beams as a function of angle (only plane waves that belong to the image portions of the beams, i.e., $\Omega_{A}, \Omega_{B}, \Delta_{A}, \Delta_{B}$, are shown). The left-hand and the right-hand figures illustrate the input and the output beams at $z=0.5 \mathrm{~cm}$ and $z=0 \mathrm{~cm}$, respectively. The angles are measured from the forward $c$ axis. 
and the intensity transmission are defined as in Ref. 1. We can obtain similar functions for beam $A$ by interchanging $\left(a_{i}\right.$ and $\left.b_{i}\right),\left(\Omega_{A}\right.$ and $\left.\Omega_{B}\right),\left(\Delta_{A}\right.$ and $\left.\Delta_{B}\right)$, and $(z=L$ and $z=0$ ) in Eqs. (15)-(17). Note that the reflectivity of one of the beams becomes equivalent to the transmission of the other when the two beams have equal intensities.

The fidelity of a phase conjugator is best measured by the conjugation fidelity, which is defined by

$$
\mathrm{CF}=\frac{\int_{-\infty}^{\infty} \mathrm{d}^{2} r A B}{\left(\int_{-\infty}^{\infty} \mathrm{d}^{2} r|A|^{2} \int_{-\infty}^{\infty} \mathrm{d}^{2} r|B|^{2}\right)^{1 / 2}},
$$

where integrations are taken over the transverse dimension $r$. Equation (17) gives the conjugation fidelity in terms of a series of discrete plane-wave amplitudes. Note that, in general, the fidelity is a complex number and that the perfect conjugation is realized when the conjugation fidelity is equal to 1 . We consider the conjugation to be complete when the amplitude of the fidelity is 1. Given that the conjugation is complete, the phase of the fidelity (fidelity phase) is equal to the bias phase acquired by the conjugated beam. We find that, for good conjugation (high fidelity values), the fidelity phases of the two beams are equal. Here we do not present a detailed study of the fidelity phase but only report its existence in the results of our calculations. In this paper the fidelity corresponds to the amplitude of CF defined in Eq. (17) unless otherwise specified.

Figure 4 illustrates the temporal evolution of the fidelity and the reflectivity for one of the beams. Notice that each function increases monotonically to its steadystate value. We define the response times of the reflectivity and the fidelity as the time for the corresponding function to reach a value that is equal to $\left(q_{f}-q_{i}\right)(1-$ $1 / e)-q_{i}$ where $q_{f}$ and $q_{i}$ are the final and the initial values of the function, respectively. In Fig. 4 the response times for the two functions are shown by vertical lines. Note that the response time of the intensity transmission for beam $\mathrm{A}$ is equal to the response time of the reflectivity for beam $B$.

Using the above figures of merit (conjugation fidelity, conjugation reflectivity, intensity transmission, and the corresponding response times), we investigate the dependencies of the DPCM on different parameters. We find that the DPCM strongly depends on $\gamma_{0} L$ (coupling coefficient times length product, or gain), where $\gamma_{0}$ is the coupling coefficient between the central spatial components of the two beams. In particular, we find a gain threshold (transition point) above which the DPCM behaves like an oscillator.

\section{CONJUGATION REFLECTIVITY VERSUS CONJUGATION FIDELITY}

In this section we establish the fact that the conjugation fidelity and the conjugation reflectivity have significantly different dependencies on gain. Here we find a transition interval (gain threshold) in which the characteristics of the solutions change dramatically. We find that the differences between the conjugation fidelity and the conjugation reflectivity are large near this transition point.
Figure 5 shows the steady-state conjugation fidelities (for both beams) and the conjugation reflectivity as functions of gain. Here the two beams possess different phase and amplitude information (see Figs. 2 and 3) but have equal total intensities. The ratio of seed intensity to beam intensity is $10^{-6}$. In this case the reflectivity and its time response are symmetric for the two beams, but the conjugation fidelity is symmetric only when the fidelity is near 1, indicating a different time evolution for the conjugated beam fidelities. Notice that the conjugation fidelity as a function of gain exhibits a sharp jump, which we define as the DPCM gain threshold, whereas the conjugate reflectivity increases smoothly as the gain is increased past that point. Also notice that near threshold the reflectivity continues to be small (although a sudden increase in reflectivity is also apparent near the transition point), whereas the fidelity is near unity, as illustrated in the experimental results in Ref. 10 .

The difference between the two functions is also apparent in the temporal evolution of the functions. Figure 4 shows the temporal evolution of the fidelity (lighter curve) and the reflectivity (heavier curve) for one of the beams (for the gain value of 1.6; threshold value is approximately 1.25). Note that the fidelity reaches a steady state sig-

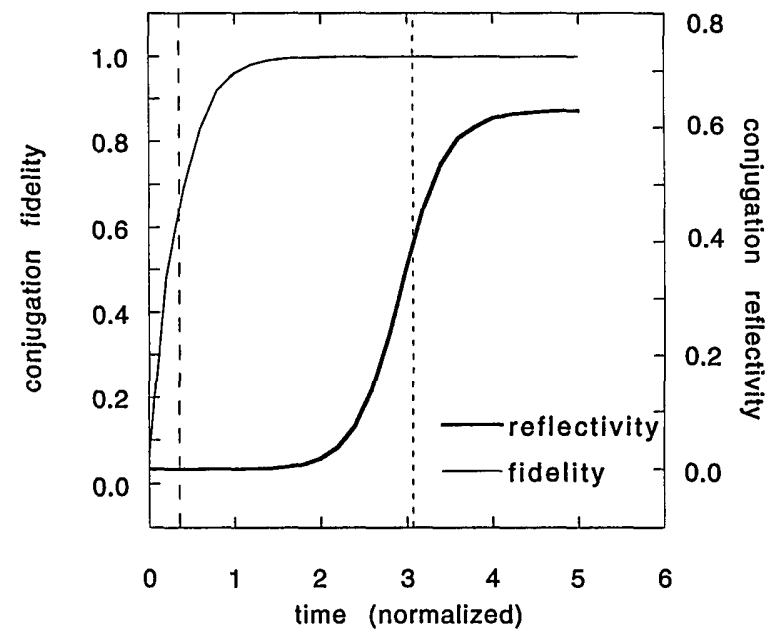

Fig. 4. Conjugation reflectivity and conjugation fidelity as functions of time in units of dark decay time. The lines indicate the two response times: long dashed, fidelity; short dashed, reflectivity.

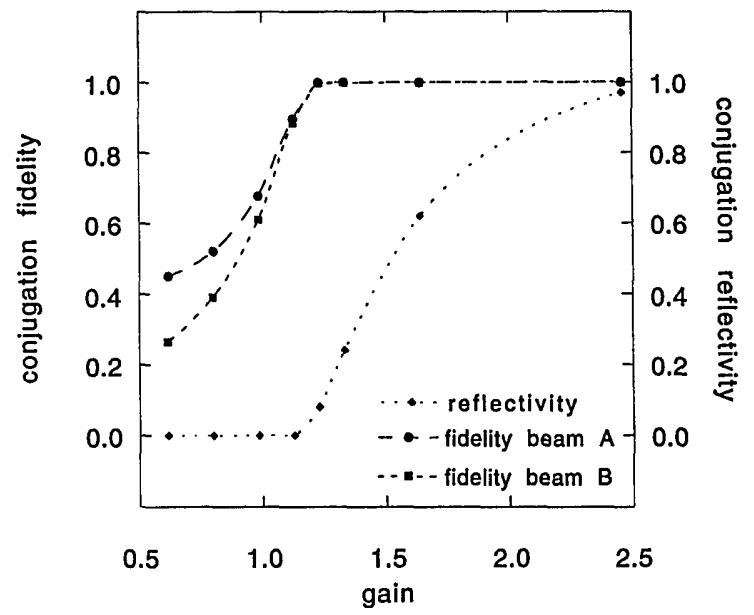

Fig. 5. Conjugation fidelities for both beams and the conjugation reflectivity as functions of gain. 


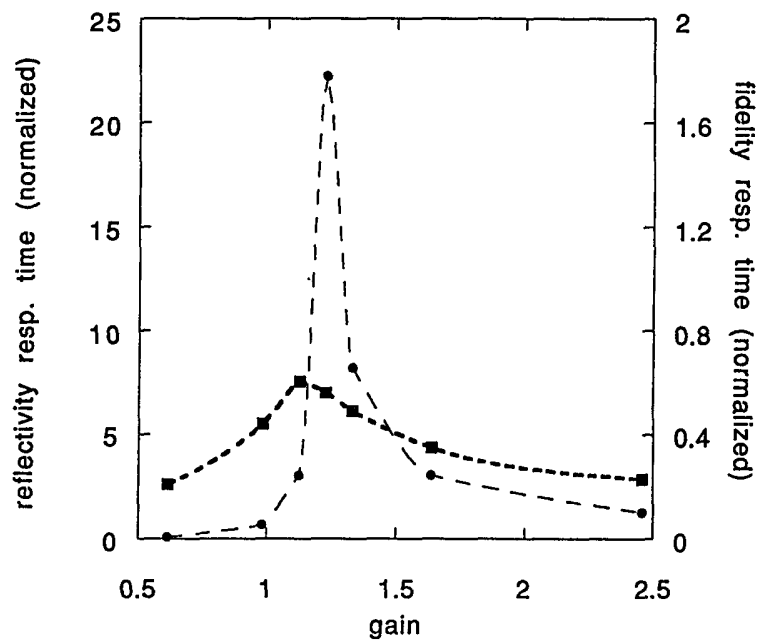

Fig. 6. Response times of the conjugation fidelity (curve with squares) and of the conjugation reflectivity (curve with points) as functions of gain.

nificantly faster than does the reflectivity. This result means that in practice the phase-conjugate beam reaches high fidelity while its intensity continues to grow in time.

\section{OSCILLATORY CHARACTERISTICS}

In this section we examine two major features that characterize the DPCM as an oscillator. The first is the slowing down of the DPCM response time for operation near threshold. Figure 6 illustrates the response times of the conjugation reflectivity and the conjugation fidelity as functions of gain. Here the beam and the seed characteristics are identical to those in the preceding sections. Note that the slowing-down effect is less pronounced for the conjugation fidelity. The difference between the two response times decreases when gain is increased. The property of decreasing response time with increasing gain is a characteristic of oscillators such as a single-mode laser. ${ }^{11}$ For an amplifier, the time response does not diverge at any specific gain value. Note that critical slowing down for PR four-wave mixing with cross-polarized pump beams was theoretically predicted ${ }^{17}$ (although not observed) for an externally pumped phase-conjugation geometry with very small signal inputs.

The second major feature that we observed is that the conjugation reflectivity is sustained after the seed levels are set to zero (for gain values above threshold). The behavior of the DPCM for different gain values is illustrated in Fig. 7, in which the temporal evolution of the conjugation reflectivities for three different gain values is shown [well above, well below, and near threshold (1.63, 0.98 , and 1.28 , respectively)]. In each case, after the system reaches the steady state the seed levels are set to zero. Above threshold the conjugate reflectivities are not affected (upper curve), whereas well below threshold the conjugate reflectivities go to zero. Near threshold the reflectivity decreases but remains finite. In these calculations we find that the conjugation fidelities improve significantly (go to unity) for all the gain values after the seed level is set to zero. For gain values below threshold this effect may be considered an artifact or a transient phenomenon because the reflectivities vanish (become small). We interpret this result as a mani- festation of a competition mechanism between selfscattering (fanning) and mutual scattering (the DPCM). Near threshold (where the reflectivities are small) fanning becomes more dominant, resulting in degradation of the conjugation fidelity. When we set the seed levels to zero (as we did previously), the fanning disappears, and we observe a dramatic increase in the conjugation fidelity.

\section{INPUT INTENSITY RATIO AND SEED LEVEL}

In this section we first examine the DPCM dependence on the seed level, as suggested by A. Zozulya (University of Colorado, Boulder, Boulder, Colorado 80309). Obtaining the results given below, we keep the information content (phase, amplitude) of the beams and the phase profile of the seed constant and vary only the amplitude of the seed. The information content of the beams is the same as in Figs. 2 and 3.

When the amplitude of the seed levels is increased, the threshold characteristic curves of the DPCM flatten. Figure 8 illustrates the fidelity as a function of gain for three different seed levels (with seed/beam intensity ratios of $10^{-2}, 10^{-3}$, and $10^{-6}$ ). Notice that for larger seed

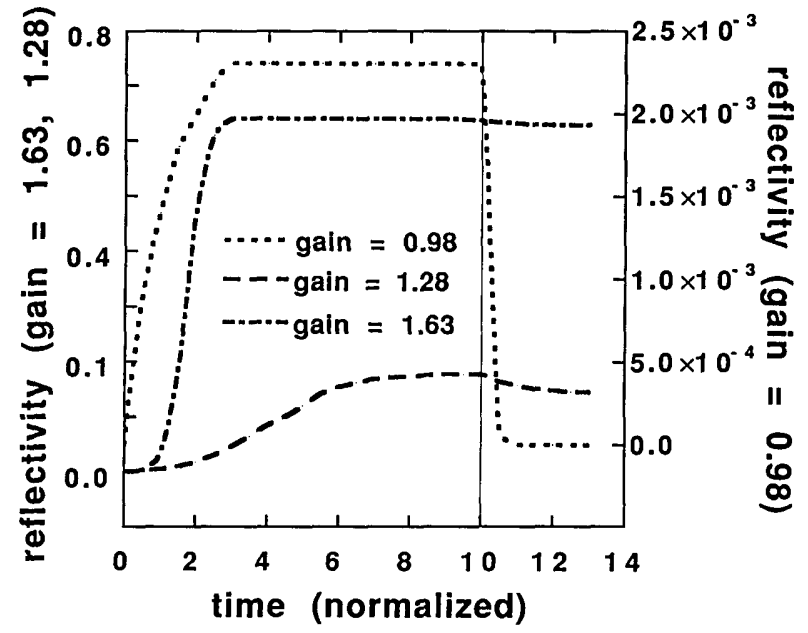

Fig. 7. Conjugation reflectivity as a function of time for three gain values for parameters that give a threshold value of 1.25.

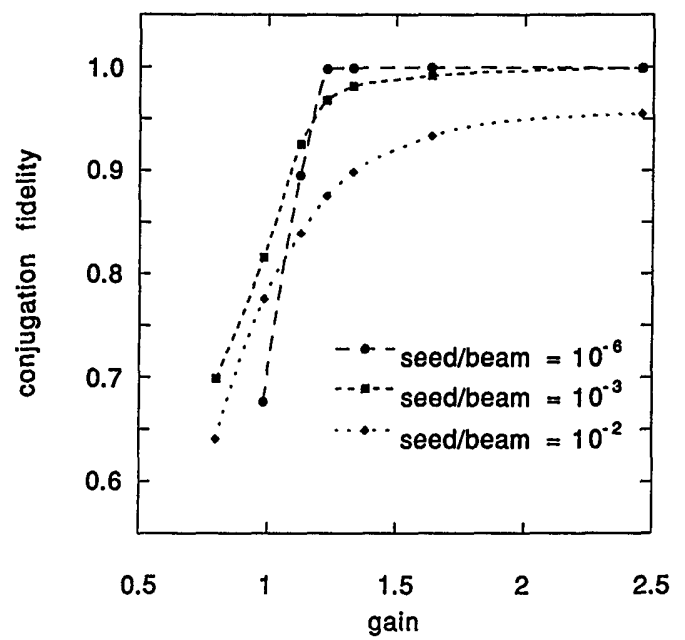

Fig. 8. Conjugation fidelity as a function of gain (for beam A only) for three different seed levels. 


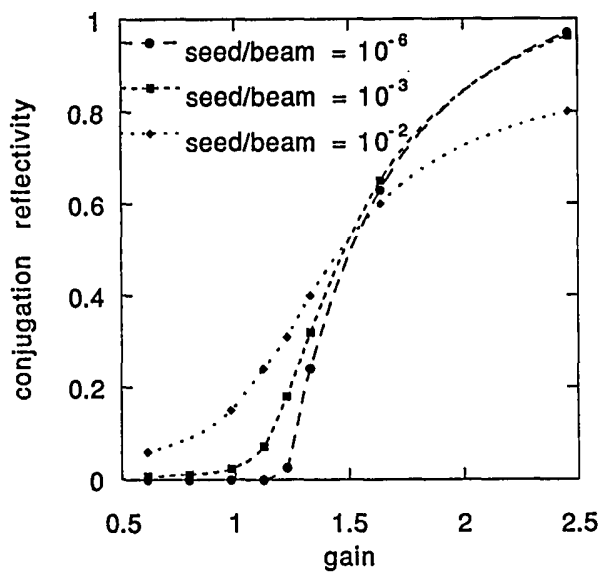

Fig. 9. Conjugation reflectivity as a function of gain for three different seed levels.

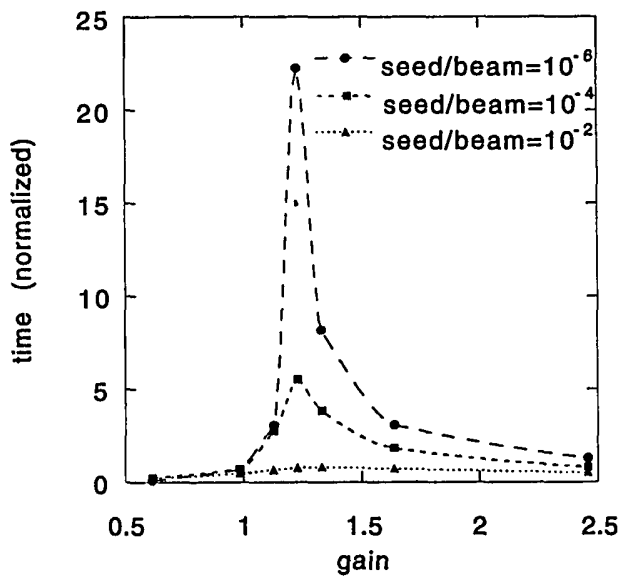

Fig. 10. Conjugation reflectivity response time as a function of gain for three different seed levels.

levels the sharp jump of the fidelity gain curve becomes less pronounced. Also notice that the degradation of the conjugation becomes significant for large seed levels. Figure 9 illustrates the reflectivity as a function of gain for the above-mentioned seed levels. The reflectivity curve becomes more discontinuous near threshold as the seed level is decreased. We again interpret these results as manifestations of the competition between the fanning and the DPCM. At low seed levels the fidelity and the reflectivity are more discontinuous near threshold, and the sharp threshold is more apparent. At high seed levels the DPCM and the fanning coexist near threshold, and as a result we observe degradation of the conjugation fidelity and smoothing of the reflectivity curve (at threshold).

Figure 10 shows the response time of the reflectivity as a function of gain for seed levels $\left(10^{-2}, 10^{-4}, 10^{-6}\right)$. Near threshold the response time of reflectivity is significantly longer for smaller seed levels. Notice that larger seed levels do not change the location of the response-time gain curve maximum, but the peak is significantly flattened. Thus critical slowing down become less pronounced as the seed level is increased. In contrast, the seed level does not affect the fidelity response time. Only at very large seed levels (with a seed/beam ratio of $10^{-2}$ ) do we observe a flattening effect similar to that discussed above. Our interpretation of this result is that the fanning acts as a dissipation process that damps the oscillation and hence shortens the response time at low gains. These dissipation effects on the fidelity response time become apparent only at large seed levels.

Variation of the intensity ratio of the two beams strongly affects the fidelity gain curves for the two beams. Figures 11 and 12 show the two fidelity curves for intensity ratios of $1,3,6$, and 9 . The conjugation is significantly asymmetric; the weaker beam is conjugated more poorly at a given gain value (i.e., the conjugation fidelity on the side of the weak input beam is greatly reduced, although the intensity of the diffracted strong beam in the direction of the weak beam is large). Notice that the conjugation fidelity on the side of the strong input beam is slightly flattened for large intensity ratios, although the threshold seems unaffected. In contrast, the fidelity on the side of the weak input beam not only is flattened but also is shifted toward higher gain values. This effect for intensity ratios other than unity was observed experimentally. ${ }^{10}$ The stronger beam bleaches the fanning gratings of the weak beam and permits relatively high conjugation quality on its input side. The opposite process, however, is inefficient. The weak beam is not able to eliminate the appearance of fanning gratings that

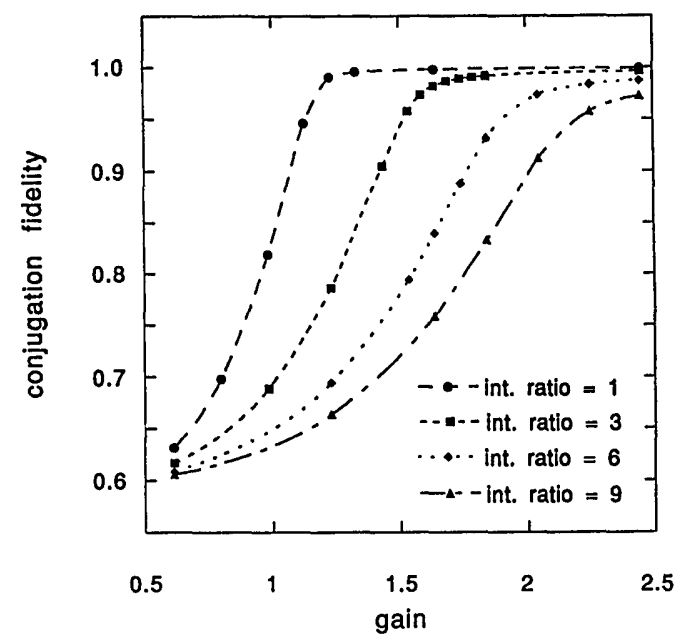

Fig. 11. Conjugation fidelity as a function of gain (strong beam) for four different input beam intensity ratios: $1,3,6,9$.

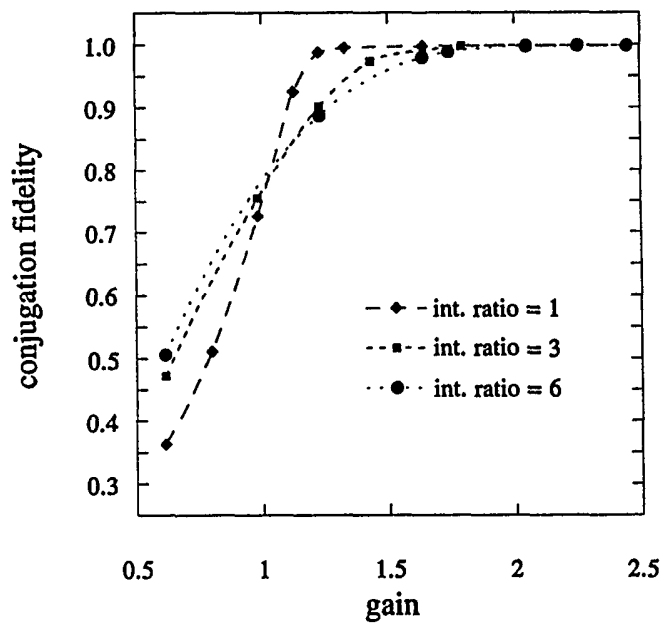

Fig. 12. Conjugation fidelity as a function of gain (weak beam) for three different input beam intensity ratios: $1,3,6$. 


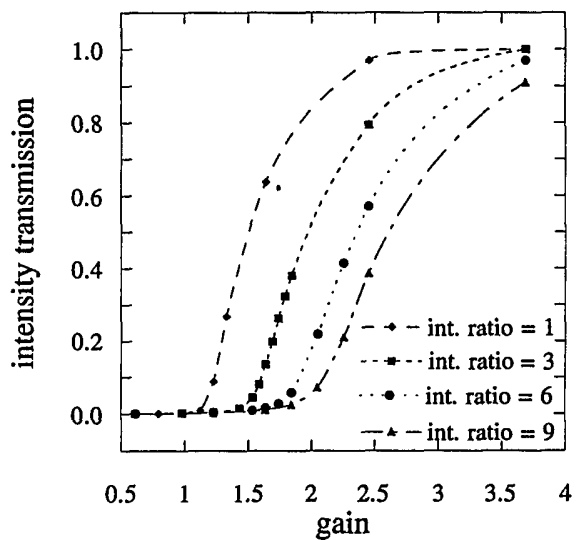

Fig. 13. Intensity transmission as a function of gain for four different input beam intensity ratios: $1,3,6,9$.

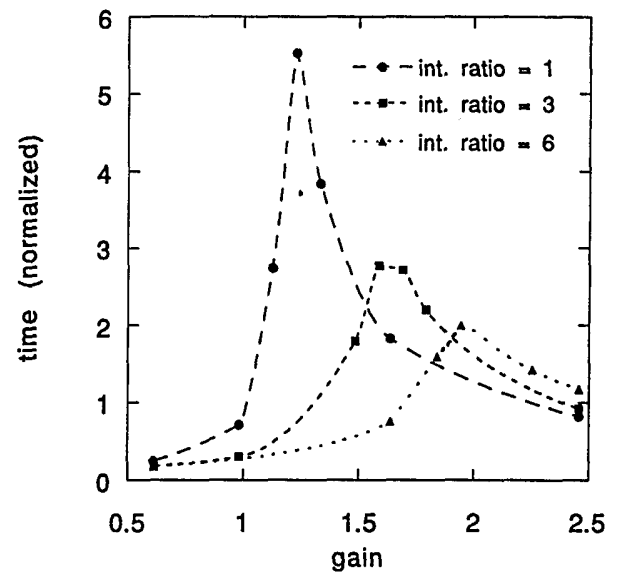

Fig. 14. Response time of conjugation reflectivity as a function of gain for three different input beam intensity ratios: $1,3,6$.

are generated by the interaction of the strong beam with the seed noise.

Figure 13 shows the transmission as a function of gain for intensity ratios of $1,3,6$, and 9 . Note that the transmission of the two beams remains symmetric. ${ }^{1}$ The reflectivity curves for the two beams can easily be obtained by multiplication of the transmission intensities with the appropriate constants (see Section 3). The result is as expected; the reflectivity on the side of the strong input beam is reduced, whereas the reflectivity on the side for the weak input beam is enhanced significantly with larger intensity ratios.

The response times of the reflectivities for the two beams remain symmetric. Figure 14 shows the response time of the reflectivity for the four intensity ratios. Note that the maximum of the curves nearly follows the threshold of the weak input beam again and that the curves are flattened for larger intensity ratios. For large intensity ratios the critical slowing down becomes less pronounced, and the fanning of the strong beam becomes more apparent. Here, once again, fanning results in the flattening of the reflectivity-response-time curve (as in the case of large seed levels). In our calculations we find that the strong input beam is conjugated significantly faster than the weak input beam (by approximately a factor of 7 near threshold), although the absolute intensity at each point in the medium is fixed. The maximum of the curve (weak input beam) nearly follows the threshold of the weak beam. We also find that the difference between two response times is larger near threshold and that the critical slowing down is much less pronounced for the stronger input beam.

\section{CONCLUSIONS}

In conclusion, we present theoretical results that analyze the double phase-conjugation process. We establish the existence of gain threshold and point out the differences between conjugation fidelity and conjugation reflectivity. We study the oscillatory nature of the DPCM and predict the existence of critical slowing down near the gain threshold. Our results permit a deeper insight into the stimulated wave mixing process and are useful for understanding the limitations of device applications, such as image processors, interconnection devices, interferometers, and coupled laser devices. Furthermore, our theoretical formulation is general and may be applied to all stimulated photorefractive processes and all self-pumped phase conjugators.

\section{APPENDIX A: DERIVATION AND REFERENCES FOR EQUATION (8)}

Here we derive the PR space-charge field as a function of the light-intensity distribution. In this respect, this calculation is the basic building block for the theory of beam propagation in PR media. Our derivation is a comprehensive expansion of the appendix given in Ref. 18.

We start from the basic equations for the PR process with no holes and no photovoltaic field ${ }^{19}$ :

$$
\begin{aligned}
\frac{\partial N^{+}}{\partial t} & =(s I+\beta)\left(N-N^{+}\right)-\gamma n N^{+}, \\
\mathbf{j} & =e \mu n \mathbf{E}+\mu k_{B} T \nabla n, \\
\frac{\nabla \cdot \mathbf{j}}{e} & =\frac{\partial n}{\partial t}-\frac{\partial N^{+}}{\partial t} \\
\nabla \cdot \mathbf{E} & =-\left(\frac{e}{\varepsilon \varepsilon_{0}}\right)\left(n+N_{A}-N^{+}\right) .
\end{aligned}
$$

Equations (A1)-(A4) are the rate equation for the density of ionized donors, the current equation, the continuity equation, and Poisson's equation, respectively. The unknowns $I, N^{+}, n, \mathbf{j}$, and $\mathbf{E}$ are the intensity profile, the ionized donor number density, the electron number density, the current density, and the electric (space-charge) field, respectively. The parameters $\beta, s, \gamma$, and $\mu$ are the dark generation rate, the cross section of photoionization, the recombination coefficient, and the mobility, respectively, and $N_{D}$ and $N_{A}$ are the total donor number density and the acceptor number density, respectively. To be consistent with the rest of the paper we use only two spatial components $(x, z)$.

We can eliminate $\mathbf{j}$ by substituting Eq. (A2) into Eq. (A3), which gives us three equations for the three unknowns $\mathrm{N}^{+}, n$, and $\mathbf{E}$. The total intensity $I$ of the two counterpropagating, mutually incoherent beams can be written with the expansions [Eqs. (5) and (6)] in the form

$$
\begin{aligned}
I(x, z, t)= & I_{0}+\sum_{m, n}^{\prime} I_{m n}(z, t) \exp [-i k(m-n) x \varepsilon \\
& \left.-i k \varepsilon^{2}\left(m^{2}-n^{2}\right) z / 2\right],
\end{aligned}
$$


where $I_{m n}(z, t)=a_{m}(z, t) a_{n}{ }^{*}(z, t)+b_{n}(z, t) b_{m}{ }^{*}(z, t)$ and $I_{0}$ is the spatial average of the intensity profile. Here $\Sigma^{\prime}$ means that the double sum is taken only over terms for which $m \neq n$. Note that the double sum has the form of the interference pattern between the spatial components of each beam. Because the two counterpropagating beams are mutually incoherent, we omit the gratings formed by their interference.

We assume similar functional forms for $N^{+}, n$, and $\mathbf{E}$ :

$$
\begin{aligned}
n(x, z, t)= & n_{0}(z, t)+\sum_{m, n}^{\prime} n_{m n}(z, t) \exp [-i k(m-n) x \varepsilon \\
& \left.-i k \varepsilon^{2}\left(m^{2}-n^{2}\right) z / 2\right],
\end{aligned}
$$

$$
\begin{aligned}
N^{+}(x, z, t)= & N_{0}{ }^{+}(z, t)+\sum_{m, n}^{\prime} N_{m n}{ }^{+}(z, t) \exp [-i h(m-n) x \varepsilon \\
& \left.-i k \varepsilon^{2}\left(m^{2}-n^{2}\right) z / 2\right],
\end{aligned}
$$

$$
\begin{aligned}
\mathbf{E}(x, z, t)= & \sum_{m, n}^{\prime} \mathbf{E}_{m n}(z, t) \exp [-i k(m-n) x \varepsilon \\
& \left.-i k \varepsilon^{2}\left(m^{2}-n^{2}\right) z / 2\right],
\end{aligned}
$$

where $N_{0}{ }^{+}$and $n_{0}$ are the spatial averages of the ionized donors number density and the electron number density, respectively, and $N_{m n}{ }^{+}, n_{m n}$, and $\mathbf{E}_{m n}$ are the Fourier coefficients of the expansions. Note that $\mathbf{E}_{m n}$, the spacecharge field component, points in the direction of the grating wave vector, $\mathbf{k}_{m n}=(m-n) \varepsilon \hat{x}+\left(m^{2}-n^{2}\right) \varepsilon^{2} / 2 \hat{z}$. In the absence of an external electric field and photovoltaic fields the spatial average of the space-charge field is zero; hence $\mathbf{E}_{0}=0$.

We substitute Eqs. (A6)-(A8) into the transport equations and separately equate terms with the same exponential factors. We assume that the electron number density is much less than the acceptor number density $\left(N_{A} \gg\right.$ $n$ ). For typical $\mathrm{cw}$ operating conditions (less than $\left.1 \mathrm{~W} / \mathrm{cm}^{2}\right)$ this assumption is well satisfied $\left(N_{A} \approx\right.$ $\left.10^{16} \mathrm{~cm}^{-3}, n_{0} \approx 10^{10} \mathrm{~cm}^{-3}\right)$. The resulting zeroth-order solutions are $n_{0}=s\left(I_{0}+I_{\text {dark }}\right)\left(N_{D}-N_{0}^{+}\right) /\left(\gamma N_{0}^{+}\right)$and $N_{0}{ }^{+}=N_{A}$, where $I_{\text {dark }}=\beta / s$.

In general, we obtain a complicated set of differential equations for the Fourier coefficients. When we omit the terms of second order in the Fourier coefficients, the set of equations becomes linear (with the above zeroth-order solutions):

$$
\begin{aligned}
\frac{\partial N_{m n}{ }^{+}}{\partial t}= & s\left(N_{D}-N_{A}\right) I_{\mathrm{dark}} I_{m n}-s I_{\mathrm{dark}}\left(1+I_{0}\right) N_{m n}{ }^{+} \\
& -\gamma\left(n_{0} N_{m n}{ }^{+}+N_{0}{ }^{+} n_{m n}\right), \\
\frac{\partial n_{m n}}{\partial t}= & \frac{-i k_{m n} \mu}{e}\left(e n_{0} E_{m n}+i k_{m n} k_{B} T n_{m n}\right)+\frac{\partial N_{m n}{ }^{+}}{\partial t},
\end{aligned}
$$

$i k_{m n} E_{m n}=\left(\frac{e}{\varepsilon \varepsilon_{0}}\right)\left(n_{m n}-N_{m n}^{+}\right)$

where we neglect terms with $\partial E_{m n} / \partial z$ and $\partial n_{m n} / \partial z$ and where $E_{m n}$ and $k_{m n}$ are the amplitudes of the corresponding vectors. Equation (9) gives a necessary condition for the validity of Eqs. (A9)-(A11). Manipulating Eqs. (A9) and (A10), we eliminate $N_{m n}{ }^{+}$and $n_{m n}$. The result is a second-order differential equation for $E_{m n}$ :

$$
\begin{array}{r}
\frac{\partial^{2} E_{m n}}{\partial t^{2}}+\left(\frac{1}{\tau_{R e}}+\frac{1}{\tau_{D m n}}+\frac{1}{\tau_{I e}}+\frac{1}{\tau_{\mathrm{die}}}\right) \frac{\partial E_{m n}}{\partial t} \\
+\left[\left(\frac{1}{\tau_{D m n}}+\frac{1}{\tau_{I e}}\right) \frac{1}{\tau_{\mathrm{die}}}+\frac{1}{\tau_{I e} \tau_{D m n}}\right] E_{m n} \\
=\frac{-i e s\left(N_{D}-N_{A}\right) I_{m n}}{\tau_{D m n} k_{m n} \varepsilon},
\end{array}
$$

where $1 / \tau_{\text {die }}=e \mu n_{0} /\left(\varepsilon_{0} \varepsilon\right), 1 / \tau_{R e}=\gamma N_{0}{ }^{+}, 1 / \tau_{D m n}=$ $k_{m n}{ }^{2} k_{b} T \mu / e$, and $1 / \tau_{I e}=s\left(I_{0}+I_{\text {dark }}\right)+\gamma n_{0}$. Here the time constants $\tau_{\mathrm{die}}, \tau_{R e}, \tau_{D m n}$, and $\tau_{I e}$ are the dielectric relaxation time, the electron-recombination time, the diffusion time, and the sum of production and ionrecombination time, respectively. For typical photorefractive crystals, $\tau_{\mathrm{di} i}$ and $\tau_{I e}$ are of the order of milliseconds, whereas $\tau_{R e}$ and $\tau_{D e}$ are of the order of nanoseconds. We also expect $E_{m n}$ to be a slowly varying function of time (of the order of seconds). Based on these physical considerations, we neglect the second derivative term relative to $1 / \tau_{R e} \partial E_{m n} / \partial t$, and $1 / \tau_{\text {die }}$ and $1 / \tau_{\text {Ie }}$ relative to $1 / \tau_{D m n}$ and $1 / \tau_{R e}$. After some algebra the differential equation takes the form

$$
\begin{aligned}
\tau_{m n} \frac{\partial E_{m n}}{\partial t}+(1+ & \left.I_{0} / I_{\text {dark }}\right) E_{m n} \\
& =-i I_{m n} / I_{\text {dark }} \frac{E_{D m n}}{1+\left(E_{D m n} / E_{q m n}\right)},
\end{aligned}
$$

where $E_{D m n}=k_{m n} k_{B} T / e$ and $E_{q m n}=e N_{A}\left(N_{D}-N_{A}\right) /$ $\left(\varepsilon k_{m n} N_{D}\right)$ are the diffusion field and the limiting space-charge field, respectively. The time constant $\tau_{m n}$ is the PR (grating) decay time at the equivalent dark irradiance and is given by $\tau_{m n}=\tau_{\text {dark }}\left(1+\tau_{R e} /\right.$ $\left.\tau_{D m n}\right) /\left(1+k_{m n}{ }^{2} / k_{d}{ }^{2}\right)$, where $k_{d}=\left[e^{2} N_{A} /\left(\varepsilon k_{b} T\right)\right]^{1 / 2}$ is the Debye wave number, $1 / \tau_{\text {dark }}=e \mu n_{\text {dark }} / \varepsilon$, and $n_{\text {dark }}$ is the dark carrier number density. Recalling the electro-optic effect, ${ }^{20}$ we obtain

$$
\delta n_{m n}=-1 / 2 n_{b}^{3} r_{m n} E_{m n},
$$

where $r_{m n}$ is an effective linear combination of the electrooptic tensor elements, which depend on the crystal orientation, the polarization of the beam, and the direction of $\mathbf{E}_{\mathrm{mn}}$. With Eq. (A14) it is a simple substitution to transform Eq. (A13) into the desired differential equation for $\delta n_{m n}$, Eq. (8). In Eq. (8) the coupling constants between the plane-wave components are given by

$$
\gamma_{m n}=(\pi / \lambda) n_{b}{ }^{3} r_{m n} \frac{E_{D m n}}{1+\left(E_{D m n} / E_{q m n}\right)} .
$$

The functional form of $r_{m n}$ and the relevant values used in this paper (for $\mathrm{BaTiO}_{3}$ ) may be found in Ref. 21. Note that $r_{m n}$ has a relatively weak dependence on the angles of propagation, whereas $E_{D m n} /\left[1+\left(E_{D m n} / E_{q m n}\right)\right]$ through $k_{m n}$ has a strong dependence ${ }^{13}$ on the difference between the angles of propagation.

\section{APPENDIX B}

In this appendix we present important numerical aspects of our calculations. We check the validity of the our calculations by doubling the integration steps in time and 


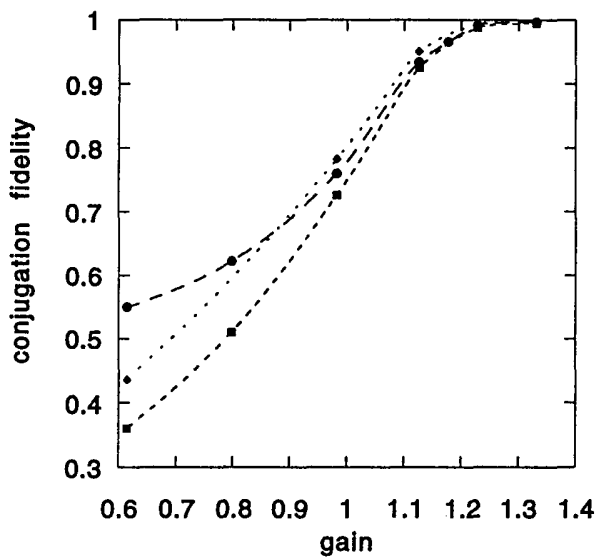

Fig. 15. Conjugation fidelity as a function of gain (for beam A) for three different pseudorandom seed phase profiles.

in $z$. In our calculations we also find that the conjugation fidelity as a function of gain below threshold strongly depends on the pseudorandom phase profile of the seed. We analyze this numerical artifact and show how we overcome it. Finally, we examine the results obtained by doubling the number of plane waves for the same angular window (see Figs. 2 and 3) and point out sampling issues.

We find that the step size of 0.05 (normalized time) in time and the step size of $10^{-3} \mathrm{~cm}$ in $z$ (the propagation direction) give results that do not change with an increasing number of steps (plane-wave amplitude is changed by less than $0.1 \%$ ). However, the above values are not necessarily optimal; optimal values vary with a number of parameters (e.g., $I_{0}$, the input intensity ratio of the two beams, etc.).

Figure 15 shows the conjugation fidelity for three different pseudorandom seed phase profiles. Notice that the differences among the three curves are large for smaller gain values (gain values below threshold). In our calculations we find that the conjugation fidelity as a function of gain strongly depends on its initial value, which can have high values for five plane waves. This dependence obscures the validity of the results, particularly for low fidelity values and for the cases in which the effects sought are small. We can overcome this limitation by using the same seed phase profile for each set of calculations or by averaging over a set of calculations with different seed phase profiles. We used the former method in our calculations. Because in practice the initial spatial correlation between the beam and the noise is expected to be small (independently of the beam characteristics), we assume that, by fixing the seed phase profile for all the calculations of a given type, we obtain the correct physical behavior.

Doubling the number of plane waves, we use interpolated values obtained from the phase and from the amplitude information of the original beam [see Figs. 2 and 3] for the additional set of plane waves. Hence the angular difference between the adjacent plane waves becomes $0.25^{\circ}$. We keep $I_{0} / I_{\text {dark }}$ fixed, and the seed/beam intensity ratio is equal to $10^{-4}$. We point out that, although all the basic characteristics that we observe (i.e., the fidelity and the reflectivity thresholds and the critical slowing down that coincide at a specific gain threshold value) persist, a shift of up to $8 \%$ of the threshold value (toward higher values) occurs for the doubled-sampling resolution case. In light of these factors, we cannot compare, within the framework of this paper, images of different fine-scale resolution borne on the input beams; we can only discuss the nature of these effects and predict their existence.

\section{ACKNOWLEDGMENTS}

This research was supported by the Advanced Research Projects Agency, the U.S. Army Office of Scientific Research, and the U.S. Air Force Office of Scientific Research. D. Engin acknowledges the financial support of the Hughes Aircraft Company, Culver City, California, through its generous Hughes Graduate Fellowship Program. We also acknowledge the supportive working environment provided by the Caltech Computing Organization at the California Institute of Technology.

*Present address, Advanced Technology Center for Photonics and Optoelectronic Materials, Department of Electrical Engineering, Princeton University, Princeton, New Jersey 08544.

\section{REFERENCES}

1. S. Weiss, S. Sternklar, and B. Fischer, Opt. Lett. 12, 114 (1987); Appl. Phys. Lett. 50, 483 (1987); Opt. Eng. 26, 423 (1987).

2. Q. B. He, J. Quantum Electron. 24, 2507 (1988).

3. Q. B. He, P. Yeh, C. Gu, and R. R. Neurgaonkar, J. Opt. Soc. Am. B 9, 114 (1992).

4. A. A. Zozulya, Opt. Lett. 16, 545 (1991); V. V. Eliseev, V. T. Tikhonchuk, and A. A. Zozulya, J. Opt. Soc. Am. B 8, 2497 (1991); N. V. Bobodaev, V. V. Eliseev, L. I. Ivleva, A. S. Korshunov, S. S. Orlov, N. M. Polozkov, and A. A. Zozulya, J. Opt. Soc. Am. B 9, 1493 (1992); K. D. Shaw, Opt. Commun. 90, 133 (1992); 94, 458 (1992).

5. A. P. Mazur, A. D. Novikov, S. G. Odulov, M. S. Soskin, and M. V. Vasnetov, J. Opt. Soc. Am. B 10, 1408 (1993) (see app.).

6. M. Segev, D. Engin, A. Yariv, and G. C. Valley, Opt. Lett. 18, 1828 (1993).

7. O. V. Lyubomudrov and V. V. Shkunov, Sov. J. Quantum Electron. 22, 1027 (1992).

8. A. V. Mamaev and V. V. Shkunov, Sov. J. Quantum Electron. 22, 1036 (1992).

9. M. Segev, D. Engin, A. Yariv, and G. C. Valley, Opt. Lett. 18, 956 (1993).

10. S. Orlov, M. Segev, A. Yariv, and G. C. Valley, Opt. Lett. 19, 578 (1994).

11. H. Haken, Synergetics (Springer-Verlag, Berlin, 1983), Chap. 5.

12. M. Born and E. Wolf, Principles of Optics (Pergamon, Oxford, 1970), Chap. 1.

13. P. Günter and J.-P. Huignard, in Photorefractive Materials and Their Applications I, P. Günther and J.-P. Huignard, eds. (Springer-Verlag, Berlin, 1989), Chap. 2.

14. M. Cronin-Golomb, B. Fischer, J. O. White, and A. Yariv, IEEE J. Quantum Electron. 20, 12 (1984).

15. Y. Fainman, E. Klancnik, and S. H. Lee, Opt. Eng. 25, 228 (1986).

16. S. Sternklar, S. Weiss, M. Segev, and B. Fisher, Appl. Opt. 25, 4518 (1986).

17. D. A. Fish, T. J. Hall, and A. K. Powell, Opt. Commun. 85, 85 (1991).

18. G. C. Valley, J. Opt. Soc. Am. B 9, 1440 (1992).

19. N. V. Kukhtarev, Sov. Tech. Phys. Lett. 2, 438 (1976).

20. A. Yariv and P. Yeh, Optical Waves in Crystals (Wiley, New York, 1984).

21. M. Segev, Y. Ophir, and B. Fischer, Opt. Commun. 77, 265 (1990). 